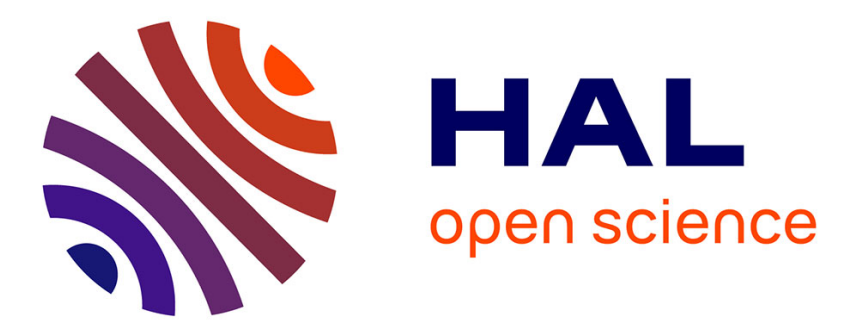

\title{
Chemistry under Vibrational Strong Coupling
}

Kalaivanan Nagarajan, Anoop Thomas, Thomas Ebbesen

\section{To cite this version:}

Kalaivanan Nagarajan, Anoop Thomas, Thomas Ebbesen. Chemistry under Vibrational Strong Coupling. Journal of the American Chemical Society, 2021, 143 (41), pp.16877-16889. 10.1021/jacs.1c07420 . hal-03448011

\section{HAL Id: hal-03448011 https://hal.science/hal-03448011}

Submitted on 24 Nov 2021

HAL is a multi-disciplinary open access archive for the deposit and dissemination of scientific research documents, whether they are published or not. The documents may come from teaching and research institutions in France or abroad, or from public or private research centers.
L'archive ouverte pluridisciplinaire HAL, est destinée au dépôt et à la diffusion de documents scientifiques de niveau recherche, publiés ou non, émanant des établissements d'enseignement et de recherche français ou étrangers, des laboratoires publics ou privés. 


\title{
Perspective
}

\section{Chemistry under Vibrational Strong Coupling}

\author{
Kalaivanan Nagarajan, ${ }^{* 1}$ Anoop Thomas*2 and Thomas. W. Ebbesen ${ }^{* 1}$ \\ ${ }^{1}$ University of Strasbourg, CNRS, ISIS, 8 allée Gaspard Monge, 67000 Strasbourg, France. \\ ${ }^{2}$ Department of Inorganic \& Physical Chemistry, Indian Institute of Science, Bengaluru, 560012, \\ India.
}

\begin{abstract}
:
Over the past decade, the possibility of manipulating chemistry and material properties using hybrid light-matter states has stimulated considerable interest. Hybrid light-matter states can be generated by placing molecules in an optical cavity that is resonant with a molecular transition. Importantly, the hybridization occurs even in the dark because the coupling process involves the zero-point fluctuations of the optical mode (a.k.a. vacuum field) and the molecular transition. In other words, unlike photochemistry, no real photon is required to induce this strong coupling phenomenon. Strong coupling in general, but vibrational strong coupling (VSC) in particular, offers exciting possibilities for molecular and more generally material science. It is not only a new tool to control chemical reactivity but it also gives insight into which vibrations are involved in a reaction. This perspective gives the underlying fundamentals of light-matter strong coupling, including a mini-tutorial on the practical issues to achieve VSC. Recent advancement of 'vibro-polaritonic chemistry' and related topics are presented with the challenges for this exciting new field.
\end{abstract}




\section{Introduction}

Nearly 100 years ago, with the advent of quantum electrodynamics (QED), it was understood that so-called spontaneous emission such as fluorescence is triggered by electromagnetic (EM) fluctuations that pervade the Universe. These EM fluctuations in turn are due to the zero-point energy of the electromagnetic states into which light emits. In other words, photons do not just fly out of molecules during the emission process but actually transit to an available electromagnetic state or mode of space. ${ }^{1}$ The higher the density of these modes, the higher the emission probability and vice-versa as shown by Karl Drexhage, a German physical chemist, in the late $1960 \mathrm{~s}^{2-3}$ by positioning fluorescent molecules above a mirror. The EM fluctuations are also responsible among other things for the spectroscopic Lamb shift ${ }^{4}$ and play a role in the van der Waals interactions.

With the above considerations in mind, if molecules are placed in an optical cavity, made for instance of two parallel mirrors, with well-defined modes, it is possible to modulate the emission probability by tuning the cavity resonance, in other words by controlling the density of optical modes at the emission wavelength. If the cavity is of sufficient quality, the emitted photons can be reabsorbed by the molecules as they bounce back and forth between the mirrors. If this exchange of photons is faster than dissipating channels, it is possible to generate hybrid light-matter states, half-electronic half-photonic. This is similar to the coupled pendula phenomenon shown in Figure 1A and is known as the strong coupling regime (Figure 1B and 1C). The formation of the two hybrid light-matter states can be easily seen in the absorption spectrum of the coupled molecule with the appearance of two new peaks (Figure 1D). Theory shows that strong coupling can be mediated by the EM fluctuations of the cavity modes, in other words it occurs even in the dark, as discussed further down. ${ }^{6}$

It should be quite intuitive to chemists that the introduction of the hybrid light-matter states, a.k.a. polaritonic states, in the ladder of the molecular energy levels should modify the properties of the molecules themselves. $\frac{6-9}{}$ Nevertheless, the chemistry of strongly coupled molecules were not given much attention until the demonstration in 2012 that it could modify the chemical landscape of the spiropyran-merocyanine photoisomerization reaction. ${ }^{7}$ This has generated considerable excitement over the past decade as a new means to engineer properties of molecules and materials. ${ }^{6,8-9}$ It has the advantage that it doesn't require a light source, as in 
photochemistry, and is relatively simple to implement even if the underlying physics can be nonintuitive.

Typically, for studies related to the molecular and material properties, either an electronic or a vibrational transition is strongly coupled to an optical cavity mode as illustrated in Figure 1B and 1C. Under electronic strong coupling (ESC) various properties and processes have been

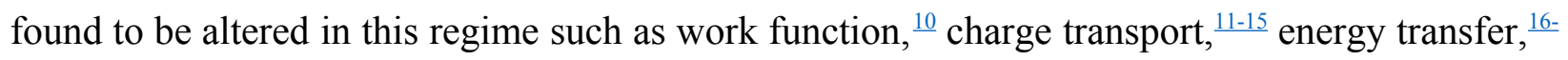
21 intersystem crossing, ${ }^{22-24}$ non-linear optics ${ }^{25-28}$ in addition to chemical reactivity., 9, 29-30 Many theoretical studies concur with the various experimental observations of modified

A)



B)

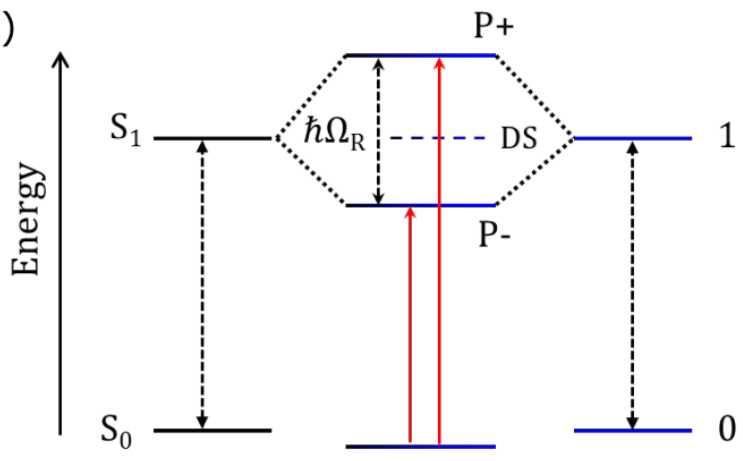

Molecular Hybrid states states EM modes
C)

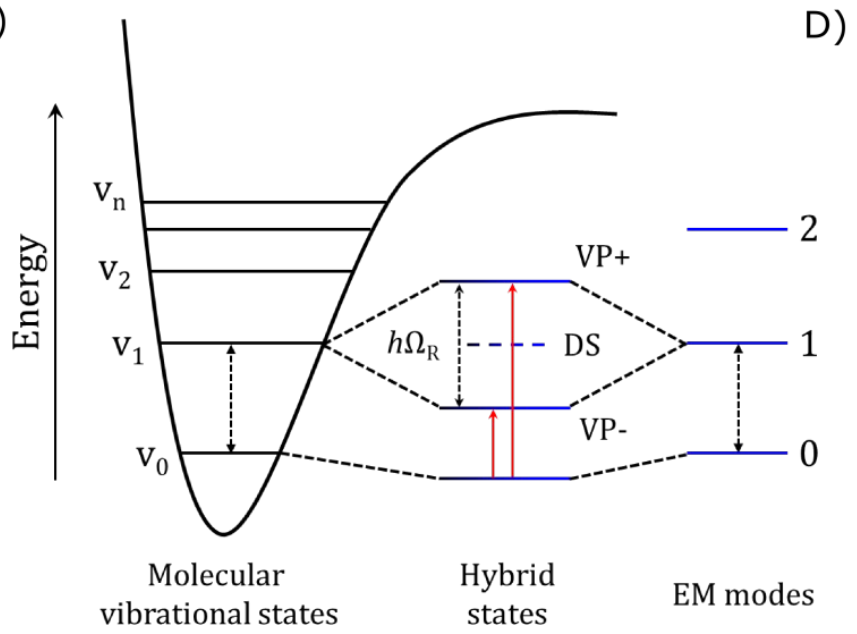

D)

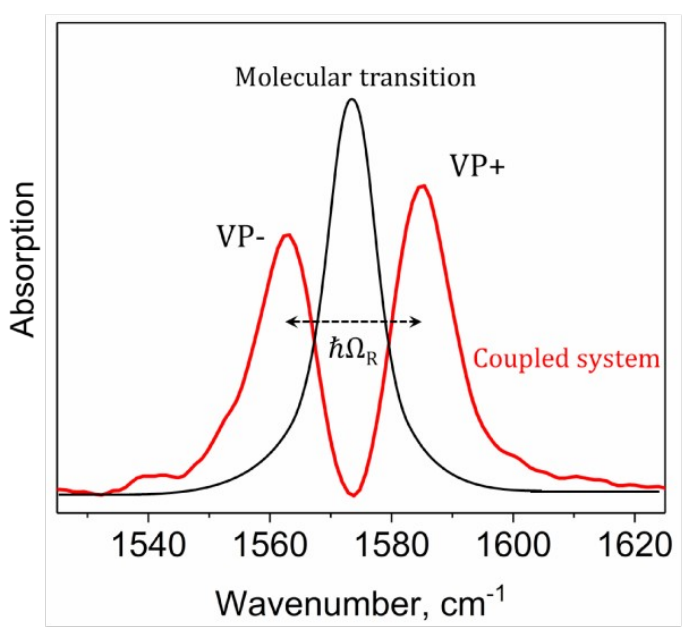

Figure 1. A) Energy exchange between the classic coupled pendula; B) Schematic representation of electronic strong coupling between an electronic transition and an optical cavity mode; C) idem for a vibrational transition; D) Absorption spectra showing the presence of VP+ and VPpolaritonic states under strong coupling (in black the initial molecular absorption and in red the coupled system). 
material properties under ESC and have provided insight into the underlying mechanisms and suggested new directions. $\frac{31-48}{}$

From a molecular science point of view, the consequences of vibrational strong coupling (VSC) has proven to be the most surprising development in this field. VSC results in the formation of vibro-polaritonic modes (VP) (Figure 1C). ${ }^{9}$ The demonstration that it could lead to large modification in chemical reactivity ${ }^{49}$ spurred much interest and stimulated many other

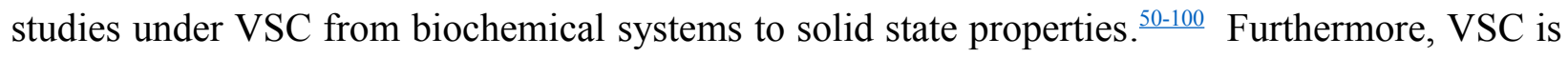
not only a new tool to control chemical reactions but also gives insight into which vibrations are involved in a reaction. To date, VSC has been shown to either accelerate or hinder the chemical reactivity of molecules. ${ }^{49,61-63,76-77,85-88}$ The possibility of coupling the reactants indirectly through their overlap with the vibrational modes of surrounding molecules (such as the matrix or the solvent), the so-called cooperative strong coupling, opens up new possibilities to modify material properties when direct coupling is not possible as explained further down. ${ }^{62,65,72,88,96,100}$ Many theoretical groups are working on this field to understand why VSC leads to such large changes in experimental observations and not surprisingly, symmetry is a fundamental factor. This perspective introduces fundamental concepts before highlighting the recent advancements of VSC to alter the chemical reactivity and molecular processes. It also provides the basic technical details to get started on this promising topic.

\section{Physics of Strong Light-Matter Coupling}

Classical pendula are coupled by the exchange of mechanical energy and thereby two new modes are formed, one where they move in phase and the other out-of-phase, as illustrated in Figure 1A. In a similar way, an optical resonator and a molecular transition are both treated as oscillators that can exchange energy (Figure 1B and 1C). Here the energy exchange is mediated by photons that can be either real or virtual. A virtual photon is an ephemeral electromagnetic fluctuation which in the optical resonator is the zero-point energy of the optical mode. It leaves no energy trace, otherwise it would violate energy conservation, however it can still mediate processes such light-matter strong coupling. 
In general, the strength of this coupling depends on the frequency of energy exchange (known as the Rabi frequency $\Omega_{\mathrm{R}}$ ) between the material transition $\left(\omega_{\mathrm{m}}\right)$ and the cavity mode $\left(\omega_{\mathrm{c}}\right)$ (Figure $2 \mathrm{~A}$ ). When $\Omega_{\mathrm{R}}$ is slower than the rate of dissipation of the material $(\gamma)$ or the cavity ( $\kappa$ ), the interacting oscillators are said to be in the weak coupling regime. In this regime, the radiative properties of the matter are modified which is also known as the Purcell effect. ${ }^{2-3,101}$ If the density of optical states is increased, the emission rate and quantum yield go up and vice-versa. In other words, the emission of a molecule is not just an internal property of the molecule itself but also depends on the optical environment in which it is located, as first demonstrated by Drexhage. ${ }^{2-3}$

When the energy exchange is faster than the dissipation channels $(\gamma$ and $\kappa)$, then the system can enter into the strong coupling regime where hybrid light-matter states are formed as shown in Fig. 1B and 1C. The most common theory for describing strong coupling is the JaynesCummings model, in which the material is considered as a two-level system resonant with a single optical mode. Hybridization between the molecular excited state and cavity photon state leads to the formation of hybrid states, $\mathrm{P}+$ and $\mathrm{P}-$. The formulation is very similar to that of Jand $\mathrm{H}$ - aggregates. $\frac{102}{}$ The newly formed hybrid states are given by

i

i

where, $i g>i$ and $i e>i$ represent the ground and excited state of the molecule respectively and $i 0>i$ and $i 1>i$ the equivalent for the cavity; $\alpha$ and $\beta$ are coefficients. According to the JaynesCummings model, in the absence of energy losses, the energy difference between the hybrid states, the Rabi splitting energy for a single molecule is given by,

$$
h \Omega_{R}=2 \sqrt{\frac{\hbar \omega}{2 \epsilon_{0} v}} \cdot d \cdot \sqrt{n_{p h}+1}
$$

where $\hbar \omega$ is the cavity resonance or molecular transition energy, $\epsilon_{0}$ the vacuum permittivity, $v$ the mode volume, $d$ the transition dipole moment of the molecule and $n_{p h}$ the number of photons involved in the coupling. Even when $n_{p h}$ goes to zero, the Rabi splitting is finite (vacuum Rabi splitting energy) due to the interaction with zero-point EM fluctuations, also known as vacuum field. In typical experiments that uses relatively large volume $\left(\sim \mu \mathrm{m}^{3}\right)$ cavities, it is challenging to achieve strong coupling with single molecule or atom, as there are more 
chances for the emitted photon to escape the cavity before getting reabsorbed by the molecule or atom. One way of exchanging photons faster than dissipation and achieve strong coupling is to couple many molecules to a single optical mode. This enhances the Rabi splitting as $\sqrt{N}, \mathrm{~N}$ being the number of coupled molecules. Such collective coupling results in N-1 dark states (DS) in addition to the two bright states P+ and P-. DS are sometimes confused with the uncoupled molecular state because both are assumed to have the same energy. This is not necessarily the case when the entropy of the DS is considered. ${ }^{74}$ In addition, they contain one excitation and are all collective delocalized states which has potentially consequences on material properties.

Collective strong coupling, whether ESC or VSC, results in the formation of coherent states that extend over micrometers in the case of ESC as observed by the in-phase fluorescence of strongly coupled molecules. ${ }^{103-104}$

While achieving strong coupling is greatly facilitated by collective coupling, it is not always possible to reach this regime either because the vibration to be coupled has weak oscillator strength or because of the limited solubility of the active molecules in the solvent of choice. To overcome this problem, the so-called cooperative coupling was developed for vibrational strong coupling (VSC). ${ }^{62,72}$ In this case, the active solute molecules are dissolved in a solvent or a matrix that has a strong vibrational band that interacts and overlaps with a vibrational mode of the solute. When the solvent vibration is under VSC, all the solvent molecules surrounding the solute are vibrating in-phase which in turn drive and couple the solute vibration.

It is important to note that the dissipation dynamics of coupled molecules is not given by the sum of the dissipation rates $\gamma$ and $\kappa$. If it was the case, the lifetime of $\mathrm{P}+$ and $\mathrm{P}$ - would be the same which is contrary to experimental observations. ${ }^{54,105}$ A so-called reservoir of uncoupled molecules has been used to account for the observed long-lived P- state, in other words, excited uncoupled molecules transfer energy to P- which then decays. ${ }^{9}$ However, P- emission quantum yield measurements demonstrates unequivocally that P- can be intrinsically long-lived, and sometimes even longer than the uncoupled molecules. ${ }^{105}$ Strong coupling also induces large unexpected changes in the chemical dynamics and potential energy surface, and hence the thermodynamics. As a consequence, coupled molecular systems must be understood as being in 
A)

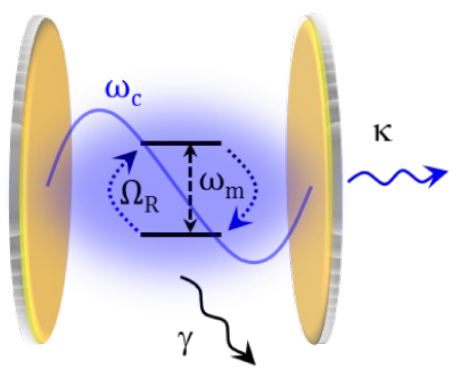

C)

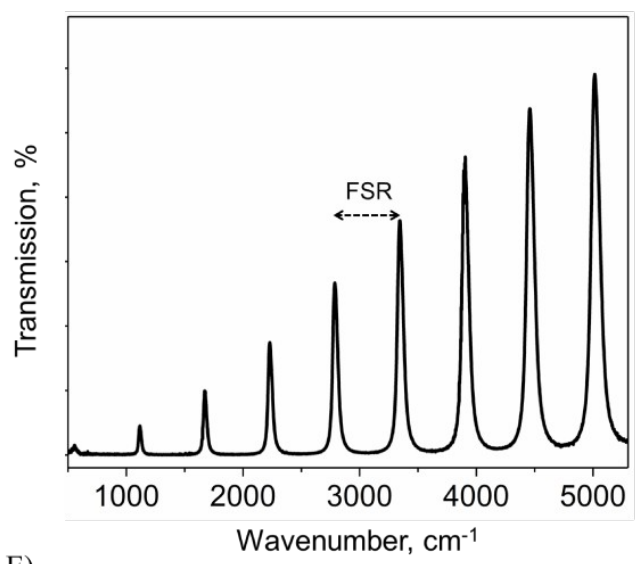

E)

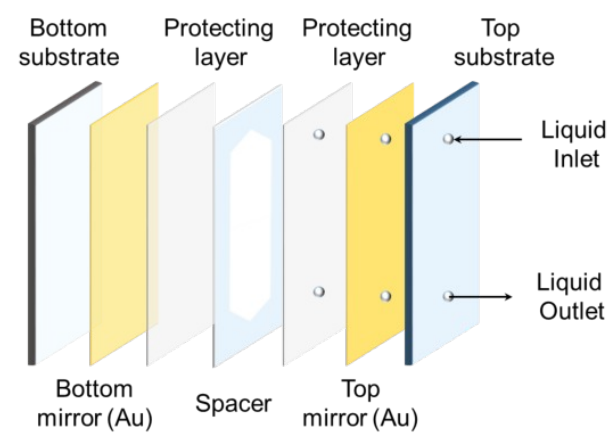

B)

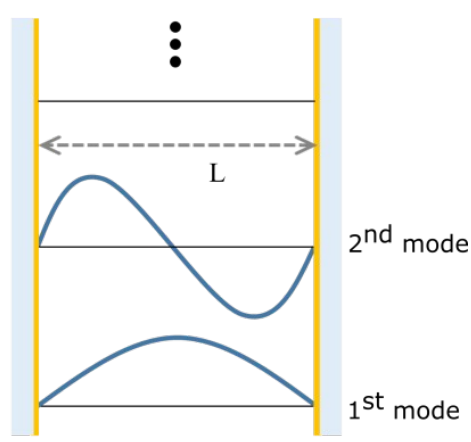

D)

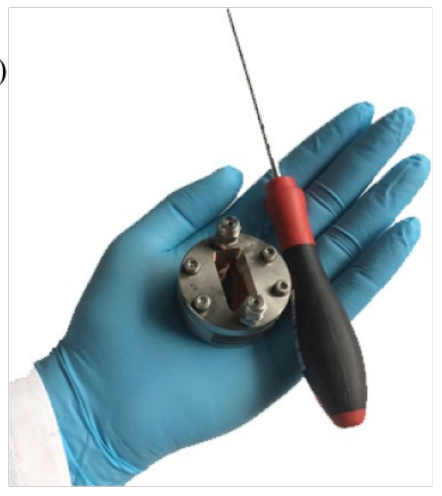

F)

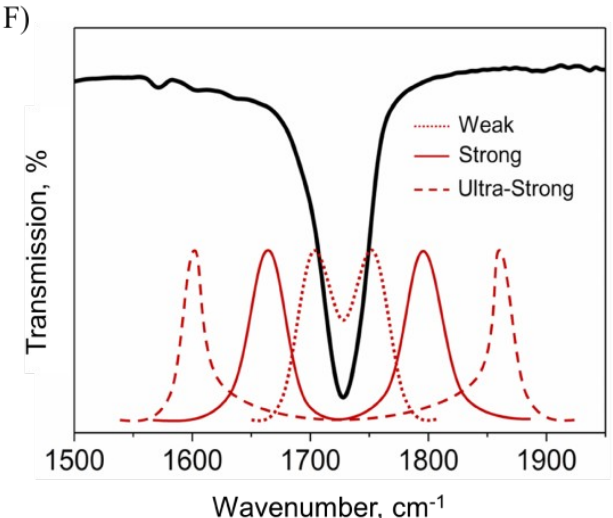

Figure 2. A) Sketch of a Fabry-Perot cavity with a mode of frequency $\omega_{c}$ and molecular transition of frequency $\omega_{\mathrm{m}}$, the coupling strength $\Omega_{R}$ and the cavity and molecular loss rates, $\kappa$ and $\gamma$, respectively; B) Sketch of the standing waves in the Fabry-Perot cavity; C) FTIR transmission spectrum of an empty (air filled) cavity of path length $6 \mu \mathrm{m}$. D) A photograph of the microfluidic Fabry-Perot optical cavity used for VSC experiments; the screwdriver is used to fine tune the cavity path length to achieve the correct resonances (courtesy of T.W. Ebbesen). E) a schematic representation of the typical Fabry-Perot cavity set up used for solution state VSC chemistry experiments, showing all the constituents and their arrangement and F) Representation of weak, strong and ultra-strong coupling conditions in the VSC regime. The black solid line represents the vibrational mode of the molecule and the red lines (solid, dotted and dashed) shows the coupled systems. The coupling is considered strong if the $\hbar \Omega_{R}$ is larger than the 
FWHM of both the molecular transition and the cavity mode, and, weak if the splitting is smaller.

the so-called non-Markovian regime where properties can only be predicted in the coupled basis. ${ }^{6,106}$ Finally suppression of spin-decoherence in strong coupled systems is also explained by a non-Markovian regime. $\frac{107-108}{}$

As the Rabi splitting $\left(\hbar \Omega_{\mathrm{R}}\right)$ increases, it starts perturbing the other states of the coupled molecules. $\frac{109}{}$ From this, the notion of ultra-strong coupling has emerged whereby the ground state acquires significant photonic character and therefore new properties. ${ }^{55,110}$ Typically, the dividing parameter is quantified by $\eta$, the ratio of the coupling strength and the energy of the molecular transition $\left(\omega_{\mathrm{m}}\right)$. When $\eta>0.1$ the system is considered in the ultra-strong coupling regime. In extreme cases, multiple Rabi splitting appears as shown for instance in the case of $\mathrm{VSC} \cdot \underline{55}$

Next, we consider the practical technical issues to achieve strong coupling.

\section{Technical Aspects of Strong Coupling Experiments}

Strong coupling experiments are quite easy to undertake but it requires some basic notions of optics and precautions in the preparation and the characterization of the samples. The confined EM field necessary for light-matter string coupling can be either provided by surface plasmons, $\frac{111-112}{2}$ an optical cavity such as a Fabry-Perot (FP) or distributed Bragg reflector mirrors (DBR). ${ }^{57}$ Obtaining high quality surface plasmon modes in the IR is not easy due to their dissipative nature, nevertheless there are examples of VSC of polymer films placed on mid-IR grating structures. ${ }^{113}$ DBRs and gratings are difficult to fabricate, relative to an FP cavity, and are less than ideal to hold liquids. For VSC of liquids and chemical reactions in solution, which is the focus of this perspective, FP cavities are the most commonly used.

\section{The Fabry-Perot cavity}

A Fabry-Perot cavity is an optical resonator which consists of two parallel highly reflecting mirrors separated by a distance L (Figure 2B). This interferometer generates a series of resonances (Figure 2C) whose wavenumbers ( $\dot{v} \dot{i}$ are given by eq. (2): 


$$
\dot{v}_{m}=\frac{10^{4}}{2 n L} m
$$

where $\mathrm{m}$ is the mode order, $\mathrm{n}$ is the refractive index of the medium, and $\mathrm{L}$ is in micrometers. The so-called free spectral range (FSR) is its value for $\mathrm{m}=1$.

For VSC, the spacing of the mirrors is typically a few micrometers $(2 \mu \mathrm{m}$ to $30 \mu \mathrm{m})$ to get the optical modes in the mid-IR region where the molecules exhibit vibrational transitions. The quality factor $\mathrm{Q}$ of the cavity is given by the ratio between the frequency of the mode and its full width half maxima (FWHM). So, the narrower the optical mode, the better will be the quality of the cavity and therefore the field confinement. The FWHM of the cavity mode is an important parameter for strong coupling since it is proportional to the cavity losses.

For the purpose of liquid phase VSC experiments, typically a commercial microfluidic FTIR flow cell is modified by coating the window substrates with metallic mirrors as explained further down with the essential components shown in Figure 2E. Substrates are flat polished IR transparent windows such as $\mathrm{ZnSe}, \mathrm{BaF}_{2}, \mathrm{CaF}_{2}, \mathrm{NaCl}$, and in some cases $\mathrm{Si}$, diamond or quartz, on which highly reflecting mirrors are coated. The selection of the window material is based on the desired transparency in the visible and the IR regions. The window substrates need to be thoroughly cleaned before mirror coating to remove the possible organic and inorganic impurities from its surface. Trace impurities may make the metal mirror non-adhesive to the window surface. Highly reflecting mirrors are essential components of optical resonators but at the same time the mirrors should be transmitting a little, enough to allow sufficient light to pass through the cavity in order to study the material inside. Mirrors made with silver (Ag) and gold $\mathrm{Au})$ are excellent IR reflectors and they can be easily deposited by sputtering on any substrate. For example, $10 \mathrm{~nm} \mathrm{Au}$ will have nearly $85 \%$ reflection in the mid IR region and the resulting cavity is good enough (Q factor $\sim 60$ ) for the VSC experiments.

The thin metal mirrors can be attacked by the molecules under study or induce chemical modifications, so a thin protecting layer is essential to avoid such problems. The mirrors are therefore typically coated with a $\sim 100 \mathrm{~nm}$ thick inert layer such as silicon dioxide, alumina or an organic polymer. $\mathrm{SiOx}$ and $\mathrm{Al}_{2} \mathrm{O}_{3}$ can be deposited through sputtering whereas an organic polymer can be spin-coated. This coating should be smooth to maintain the optical quality of the cavity. The spacer, with a hollow central channel to hold the liquid, that separates the two 
mirrors can be purchased from commercial sources and are typically made of polyethylene terephthalate (Mylar), polyimide (Kapton), polypropylene and Teflon. The flexibility of the spacer helps to fine tune the cavity length $\mathrm{L}$ with the screws holding the mount together. Because the pressure of the screws slightly deforms the windows, visual inspection of the $\mathrm{cm}$ size FP cavity reveals weak interference fringes in the visible. These are used to ensure that a large central area (nearly $50 \%$ of the total area) is in tune where the property under observation, such as the chemical reactivity, is monitored. Typically, a cache with a hole is placed on the microfluidic holder to define this analyzed area. The non-uniformity of the metal/dielectric coating and small wrinkles on the spacer will affect the quality of the formed cavity which is immediately noticed from the deformed structure of the interference fringes and the reduced size of the central tuned region. An online video is available to illustrate all these issues. ${ }^{114}$ To observe the effects of strong coupling, the optical resonance and the molecular transition must be in exact resonance at normal incidence (see below). The flow cell is kept in a thermostatic jacket in order to maintain the temperature or to perform temperature dependent experiments. Note that liquids can diffuse through the spacers so very volatile solvents should be avoided as well as high temperatures. Furthermore, after the compression of the spacer, the cavity needs time to equilibrate. The stability of the cavity is easily assessed by monitoring the FP modes. Typically, a cavity requires a minimum of 20 minutes to become stable before further steps can be undertaken. This is particularly critical for VSC of narrow vibrational modes in the mid-IR region.

\section{Characterization of strong coupling}

Once the IR spectrum of the molecule to be coupled is known, the empty (air filled) cavity is also characterized by FTIR. It will show a transmission spectrum with a series of resonances. The frequency of these modes, which are multiples of the fundamental cavity mode, in wavenumber units is given by the equation 2 . For chemical reactivity studies that require cavity stability for 30 minutes or more, the best procedure is to estimate in advance the mode frequency or wavenumber that will be needed when the cavity is filled with the solution of refractive index $\mathrm{n}$ so that the cavity mode and the vibration are in resonance once filled. However, it must be noted that a strong absorption band can induce local variations of the refractive index (Kramers-Kronig relations), thereby shifting the mode position relative to that 
calculated based on the average $n$ value of the solution. The empty cavity is then tuned accordingly. The stability of the empty cavity is then assessed at the reaction temperature before injecting the material (preferably also at the same temperature).

The characterization of strong coupling, and that this regime has been achieved, involves the recording of the FTIR spectra of the cavity plus the molecules of interest to observe the split peaks and measuring their angle dependent dispersion. Split peaks are not in themselves a proof that the strong coupling regime has been attained (Figure 2F). Strong coupling is confirmed when the polaritonic peaks $\mathrm{P}+$ and $\mathrm{P}$ - are separated by an energy $\hbar \Omega_{\mathrm{R}}$ which is greater than the FWHM of both the molecular vibration and the cavity mode. The best way to see the true Rabi splitting is to measure the spectrum of the coupled system as a function of angle because the FP modes are dispersive. The dispersion is given by:

$$
k_{i \vee i=2 \pi v \sin \theta i}
$$

where $k_{\|}$is the in plane momentum; $v$, energy of the mode in wavenumbers; $\theta$, the incident angle. The two peaks will approach each other and then separate again, i.e. an avoided crossing at the molecular absorption (Figure 3). The minimum separation is $\hbar \Omega_{R}$ and only in this condition are the polaritonic states $50 \%$ photonic. It should be noted that shifts in the FP transmission peaks with angle can even occur when the system is not under strong coupling. For instance, if the vibrational band is in between two FP modes, the latter will shift with angle due to the associated index variation around the vibrational band. This can lead to erroneous interpretation of the data as indicating VSC. This problem can be avoided by knowing the FSR since the position of the FP modes are then well known and one should match that of the vibration. 


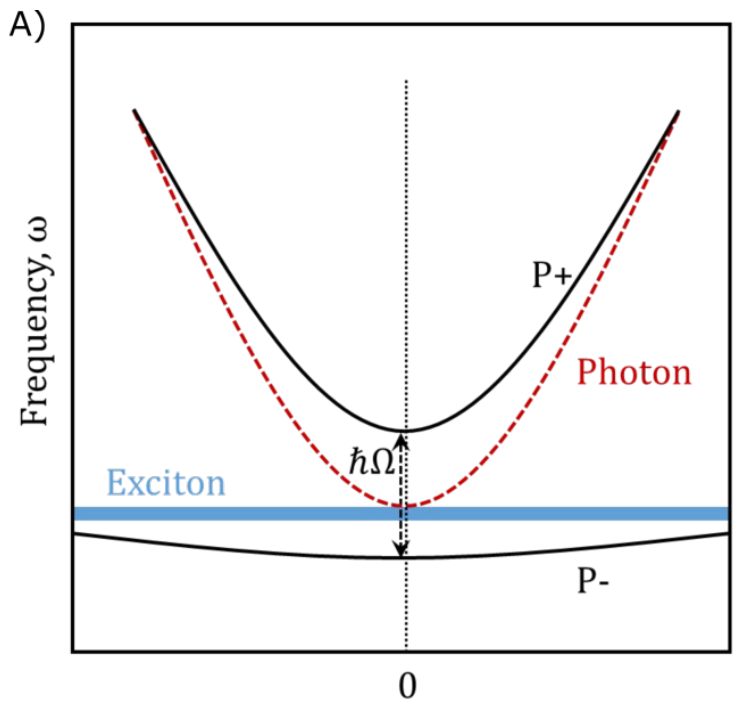

In-plane momentum, $\mathrm{k}_{/ /}$

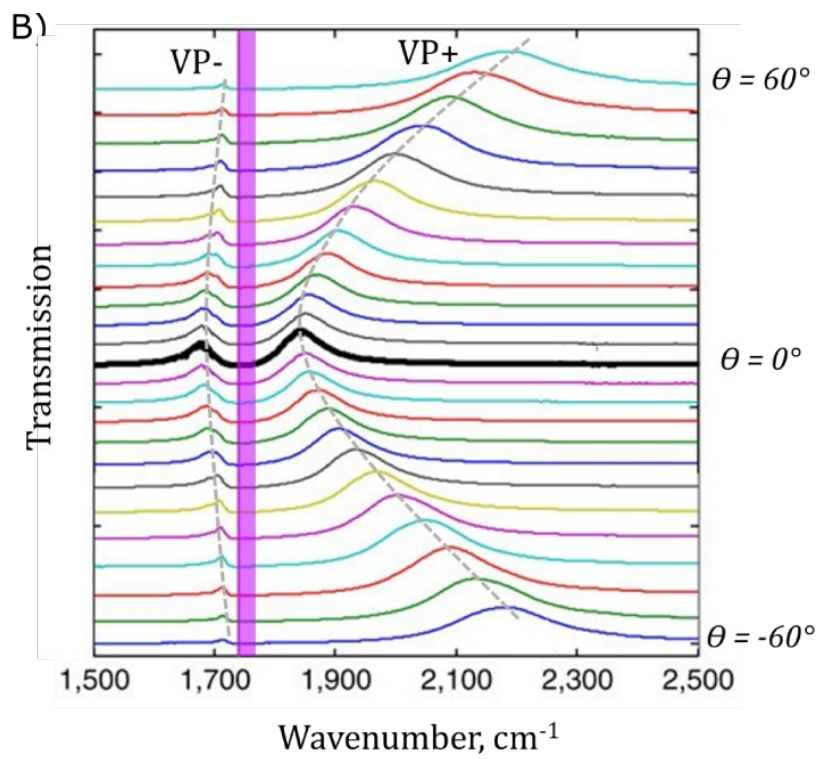

Figure 3. A) Schematic representation of a dispersion of a FP cavity tuned such that the anticrossing appears at normal incidence $\left(\mathrm{k}_{\|}=0\right)$. B) Transmission spectra of the coupled cavity system $(\mathrm{C}=\mathrm{O}$ vibration of polyvinyl acetate is coupled with the cavity mode) as a function of the IR-beam incidence angle, showing the dispersion of the cavity mode which intersects with the vibration, to generate VP+ and VP- at the minimum energy separation $\hbar \Omega_{\mathrm{R}}$ (reprinted with permission from ref. 50).

Importantly, experiments over the past decade show that to modify molecular and material properties, the strong coupling must occur at normal incidence. In physics term, strong coupling must be tuned to occur when $\mathrm{k}_{/ /}$, the in-plane momentum, equals 0 in a dispersion curve as illustrated in Figure 3. The reason for this is not well understood and only recently has two different theoretical studies also reported such a resonance effect. ${ }^{95-96}$ We speculate that when $\mathrm{k} / /$ $=0$, the coupled system is at the minima of the potential energy curve (Figure 3), in other words at the equilibrium position where it remains. When the system is coupled at another $\mathrm{k} / /$ value, it can relax away from the hybrid coupled states to the minimum in the dispersion curve by losing momentum (k) and energy to the environment.

\section{Monitoring the property in a cavity}

Measuring a property inside a cavity is not always straight-forward and many controls are necessary. For instance, the same experiment needs to be performed under off-resonance conditions where none of the cavity modes are strongly coupled with any of the molecular 
vibrations. When possible, a given property can be measured as a function of tuning across a vibrational band and if carried across several vibrations will result in an action spectrum, revealing which vibrations are involved in the measured property. $\underline{\underline{63}}$

A change in property can be monitored in the IR by following an isolated FP mode when, for instance, the reaction results in products with slightly different refractive index (eq. (2)). One should ensure that the cavity has been equilibrated at the experimental temperature before injecting the reactants to avoid any instability related shifts as discussed earlier. Alternatively, when possible, the modification can be recorded in the visible by fluorescence or absorption because the modes are typically very weak so far away from the IR tuned FP cavities, facilitating the measurements. To avoid the presence of the residual sharp small fringes in the visible, spacers larger than $12 \mathrm{um}$ should be used together with ca. $10 \mathrm{~nm}$ slit widths. To acquire the reference spectrum for absorption, it is best to reuse the same cavity used for sample measurements. This will ensure that the optical characteristics of the components of the cavity and its central tuned part remains the same for both sample and reference measurements.

It is however quite difficult to measure accurate absorption spectra of material inside a cavity in the same spectral range where the system coupled (the IR for VSC), because the cavities are highly reflective. Hence the absorption A spectrum can only be determined if both the transmission $\mathrm{T}$ and the reflection $\mathrm{R}$ are known since $\mathrm{A}=1-\mathrm{T}-\mathrm{R} \cdot \frac{115}{}$ For pump-probe experiments, it is therefore best to make one of the mirrors ca. $100 \%$ reflective and collect the light in reflection mode since then $\mathrm{A} \sim 1-\mathrm{R} . \underline{115}$

It has been suggested that surface plasmons of the FP mirrors could also contribute to VSC. $\frac{113}{1}$ If this is the case, then the contribution must be very small because no effect of VSC can be seen with FP off-resonance control experiments. This might be due to the fact that the surface plasmon field decays exponentially from the surface. The windows of the FP cavity system are so thick $(>2 \mathrm{~mm})$ that there are no well-defined slab modes that could possibly generate strong coupling conditions. $\frac{116}{}$

There is normally sufficient material even in a micro cavity to measure the $\mathrm{NMR}^{\frac{85}{5}}$ or mass ${ }^{\underline{63}}$ spectrum of the products. In the case of supramolecular assembly $\frac{100}{}$ and crystallization ${ }^{90}$ under strong coupling, microscope images can be used to monitor the changes in the morphology and crystal structure respectively. It is important to follow such an analysis in the tuned area of 13 
the cavity to see the changes under strong coupling. This allows one to measure quantitatively products and products ratios unambiguously. The yield, however, must then be corrected for the tuned area of the cavity. Control studies have shown that, in the time frame of a reaction monitored over 30 minutes to an hour, the diffusion between the tuned and detuned region is minimal in the microfluidic cells and cavities used for the experiments, ensuring the correctness of the yield. Nevertheless, the diffusion in given experimental conditions should always be checked to ensure the accuracy of the yields.

\section{Progress on Chemical Reactivity under VSC:}

The use of VSC to modify ground state chemistry was first suggested in the report on the reactivity of merocyanine - spiropyran under ESC. ${ }^{7}$ This led to the study of VSC of molecules under different conditions $\frac{51-57}{1}$ and finally to the demonstration that chemical reactivity can be indeed be modified in this regime. ${ }^{49}$ The biggest surprise of this initial chemistry study was the large change in the thermodynamics of the reaction, an order of magnitude larger than the Rabi splitting $\hbar \Omega_{\mathrm{R}}$ which is typically on the order of $\mathrm{k}_{\mathrm{B}} \mathrm{T}$, i.e. thermal energy. It also implied, among other things, that the effect of VSC was transferred efficiently to the electronic potential energy surface via vibronic interactions.

It thus raised many questions and spurred further studies both theoretical and experimental. $^{54,58-100}$ Although an unlimited number of reactions are available, so far only a dozen have been reported under VSC due to the steep learning curve to use this regime. One study reported changes in 5 different reactions ${ }^{87}$ but more recent work showed that at least 1 of those are not reproducible. ${ }^{91}$ The experiments are certainly not easy to do correctly hence the precautions indicated in the previous sections. To facilitate initial experiments, the reaction mixture should be a homogeneous liquid; the constituents should not be very volatile and since there is no provision to stir the reaction mixture in the microfluidic cells, the solutions have to be premixed before injection. With the current cavities it is difficult to follow fast reaction, therefore it is better to choose those reactions that require a reaction time of the order of 30 minutes or more. Once more sophisticated microfluidic circuits are used, faster reactions and in situ mixing can be considered. The molecules should have distinct and strong vibrational bands in order to couple them with the optical modes. After choosing the reaction to study under VSC, 14 
the strong coupling can be achieved through direct or cooperative coupling as already explained. In addition, it is best to keep the strong coupling constant for the period of observation. For instance, under cooperative VSC with the solvent, it remains constant during the reaction. Under direct coupling, it is best to run the reaction for a period such that the coupled reactant is not significantly consumed. Finally, it should be pointed out in the weak coupling regime, no changes in chemical reactivity or product ratios are observed as expected since it only modifies the density of optical states and thereby the emission probability.

Table 1 summarizes all the reaction reported to date and some we detail below.

Table 1. Summary of reactions reported under vibrational strong coupling 
Reaction Kinetics under VSC

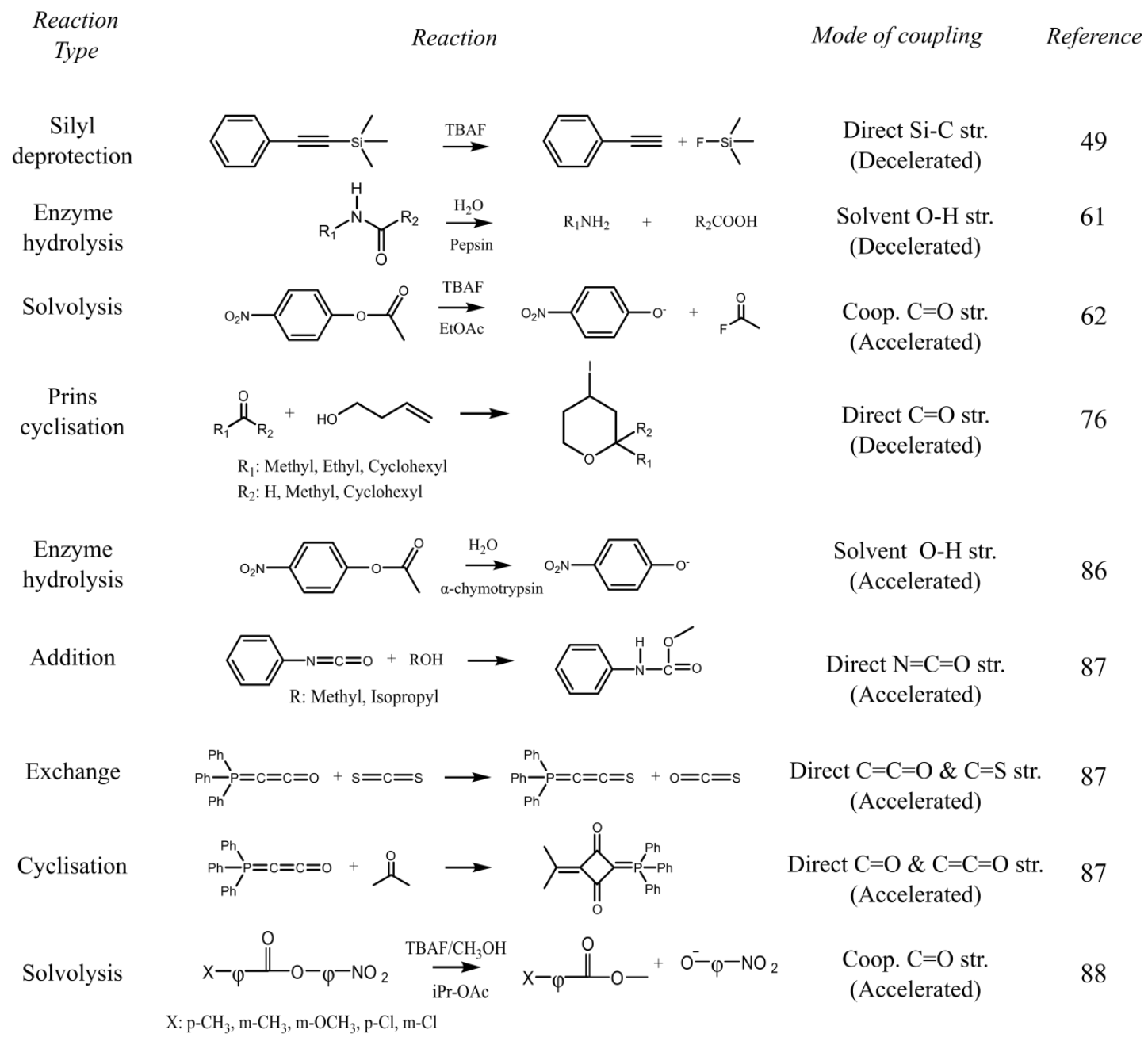

\section{Chemo-selectivity under VSC}

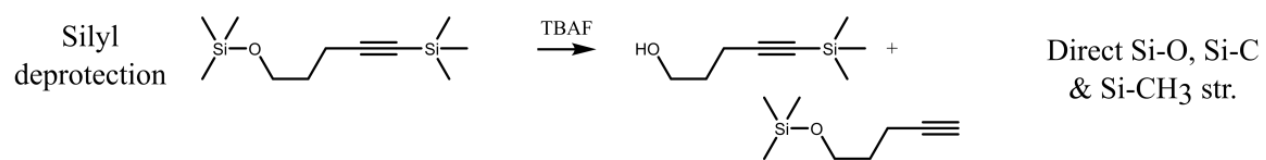

Charge-Transfer equilibrium under VSC

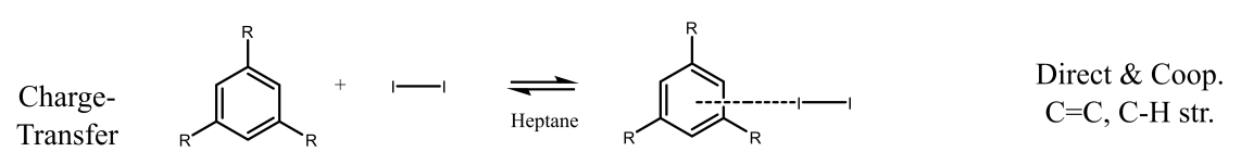

Electrocyclic ring opening R: $H$ and Methyl

\section{Stereo-selectivity under VSC}

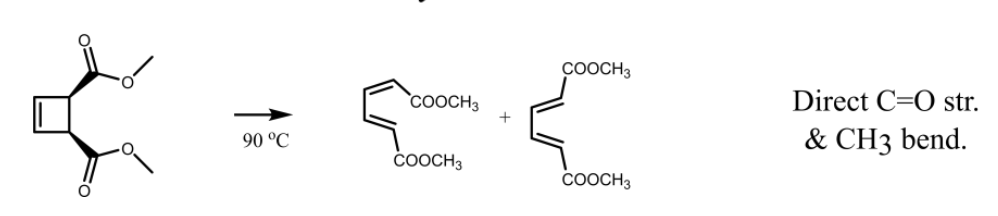




\section{Reaction Kinetics}

The very first example of chemical reaction studied under VSC was the deprotection of 1-phenyl-2-trimethylsilylacetylene (PTA) with tetra- $n$-butylammonium fluoride in methanol. ${ }^{49}$ The pseudo-first order reaction was studied by coupling the $\mathrm{Si}-\mathrm{C}$ bond of PTA and found that the reaction rate is retarded by a factor of 5.5. The kinetics was extracted from the temporal shift of cavity modes, resulting from the slight difference in the $\mathrm{n}$ of the product and the reactant, reflecting the progress of the reaction. Interestingly, the change in the reaction kinetics varies with the Rabi splitting energy, further confirming the role of VSC in the reaction. ${ }^{78}$ Thermodynamic analysis shows that the enthalpy $\left(\Delta H^{\ddagger}\right)$ and the entropy $\left(\Delta S^{\ddagger}\right)$ increase with $\Delta S^{\ddagger}$ even changing sign. This suggests that the reaction mechanism of the $\mathrm{Si}-\mathrm{C}$ cleavage is altered from an associative to a dissociative pathway under VSC. Thus, VSC can lead to surprisingly large modifications in the ground state potential energy surface. A recent theoretical study explains much of these findings and will discussed further down. ${ }^{95}$

Since this first report, other classes of reactions have been studied under VSC. For example, the Prins cyclisation of series of aldehydes and ketones with alkene slows down by a factor of $2-5$ depending on the reactant, when the $\mathrm{C}=\mathrm{O}$ stretching mode of aldehyde and ketone was coupled..$^{\frac{76}{6}}$ The $\Delta H^{\ddagger}$ increases but $\Delta S^{\ddagger}$ remains unchanged when compared to the non-cavity conditions.

In the solvolysis of para-nitrophenyl acetate (PNPA), the reaction rate increases by an order of magnitude under cooperative strong coupling of the $\mathrm{C}=\mathrm{O}$ stretch of the reactant and the overlapping $\mathrm{C}=\mathrm{O}$ stretch of the ethyl acetate solvent. ${ }^{62}$ A control reaction carried out under the VSC of ${ }^{13} \mathrm{C}=\mathrm{O}$ rich ethyl acetate $\left({ }^{13} \mathrm{EtOAc}\right)$ having non-overlapping ${ }^{13} \mathrm{C}=\mathrm{O}$ stretching mode with the reactant ${ }^{12} \mathrm{C}=\mathrm{O}$, and induces no change in the reaction kinetics, confirming the cooperative strong coupling. The kinetics were extracted from the absorption band of the product in the UV. The reaction was carried out to completion in order to obtain the final absorbance from which the kinetics could be calculated. The lowering of the barrier (both $\Delta H^{\star}$ and $\Delta S^{\natural}$ ) and the enhanced reaction rate is akin to a catalytic effect induced by the cavity coupling. The thermodynamics suggest that solute-solvent interactions are modified under VSC, further stabilizing the transition state. Recently six other para-nitrophenyl benzoate (PNPB) derivatives under cooperative VSC 
have been reported showing enhanced solvolysis rates and interestingly, the linear free energy relationship is modified under strong coupling.. .8

Enzymatic activity under VSC has been reported in two different studies. ${ }^{61,86}$ In one case the activity of protease pepsin was studied under VSC of water molecules. Water is involved at different levels in such an enzyme: in the reaction site, in the conformation of the protein and in the surrounding medium. An approximately 4.5 -fold decrease of the second-order rate constant $k_{\text {cat }} / K_{\mathrm{m}}$ was observed when coupling the $\mathrm{O}-\mathrm{H}$ stretching vibration, whereas no effect was detected for the strong coupling of the bending vibration of water. ${ }^{61}$ This observation points to the use of VSC as a mechanistic tool to understand which vibration is involved in a reaction. In the study of $\alpha$-chymotrypsin, the catalytic efficiency was enhanced 7-fold under strong coupling in the ester hydrolysis reaction of PNPA. ${ }^{86}$ The $\mathrm{O}-\mathrm{H}$ and $\mathrm{N}-\mathrm{H}$ vibrations of $\alpha$-chymotrypsin were cooperatively coupled through the $\mathrm{O}-\mathrm{H}$ stretching of water molecule. The enhancement in the enzyme efficiency under VSC was ascribed to the perturbation in the proton-transfer process which is the rate determining step in the reaction mechanism. Further studies will be necessary to understand exactly how VSC affect enzymatic activity and at what levels.

\section{Chemo-selectivity}

Observation of modified reaction kinetics of Si-C deprotection under VSC discussed above, led naturally to the question of whether VSC could modify chemo-selectivity. ${ }^{63}$ For that purpose, a molecule containing two possible reaction sites was prepared. The molecule (tertbutyldimethyl([4-(trimethylsilyl)but-3-yn-1-yl]oxy)silane) can undergo silyl bond scission at two sites, Si-O and Si-C, when reacting with TBAF. Remarkably, when the reaction is performed under VSC of selected vibrational modes $\left(\mathrm{Si}-\mathrm{C}, \mathrm{Si}-\mathrm{O}\right.$ and $\mathrm{Si}-\mathrm{CH}_{3}$ ) of the reactant, the reactive landscape tilted in favor of the Si-O bond scission and modified the branching ratio when compared to outside the cavity. This is further confirmed by the thermodynamic analysis. $\frac{63}{}$ The action spectrum of VSC as a function of cavity tuning, once again points to the use of VSC as a mechanistic tool to identify the vibrations involved in reactions.

\section{Role of Symmetry and Stereo-Selectivity}


While significant modifications in chemical kinetics and reaction pathways under VSC are being found in different labs (Table 1), there is still much to be understood about the underlying mechanism(s). Since the work of Woodward, Hoffmann and Fukui, it is well known that symmetry, and the corresponding correlation diagrams, plays a key role in defining the potential energy surface connecting reactants and products, even if other factors can obscure its effect. $\frac{117-119}{}$ In order to test whether symmetry is also an important ingredient in VSC, a wellknown charge transfer $(\mathrm{CT})$ equilibrium reaction between mesitylene and iodine was chosen. ${ }^{\text {I7 }}$ By coupling the vibrational modes of mesitylene with different symmetry, the equilibrium constant $\mathrm{K}_{\mathrm{DA}}$ of the $\mathrm{CT}$ complex was studied and it was found that $\mathrm{K}_{\mathrm{DA}}$ was increased or decreased depending on the symmetry of the molecular vibration being coupled but independent of the coupling frequency and the type of vibration. ${ }^{77}$ Similar result was observed in a charge transfer complexation between benzene and iodine under VSC. It suggests that the VSC acts on the vibrational symmetry and thereby perturbing the correlation diagram. This will naturally change the reaction barrier up and down. It also explains why a small Rabi splitting can induce such large change since VSC does not just act through the magnitude of the splitting but also on the symmetry of the vibration. In this context, molecular symmetry mediated chemical reaction such as pericyclic reaction should be affected and is indeed observed. $\frac{85}{} \mathrm{~A}$ unimolecular thermal electrocyclic ring opening of a cyclobutene derivative (cyclobutene-cis-3,4-dimethylcarboxylate (CB)), which follows Woodward- Hoffmann (WH) rules, was studied under VSC. As predicted by the WH rules, the symmetry allowed cis-trans butadiene is obtained as the major product (92\%) while the symmetry-forbidden trans-trans derivative is present only with $8 \%$ yield upon ring opening of $\mathrm{CB}$ at $90^{\circ} \mathrm{C}$ under non-cavity conditions. However, under VSC conditions with different vibrational modes of $\mathrm{CB}$, the stereo-selectivity of the reaction predicted by $\mathrm{WH}$ is modified as can be seen by the product analysis using NMR (Figure 4A). Thermodynamic analysis suggests that the transition states of the reactions leading to symmetry allowed (conrotatory) and symmetry forbidden (disrotatory) product were stabilized or destabilized (Figure 4B) by coupling the different vibrational mode of CB as expected. $\frac{85}{5}$ 

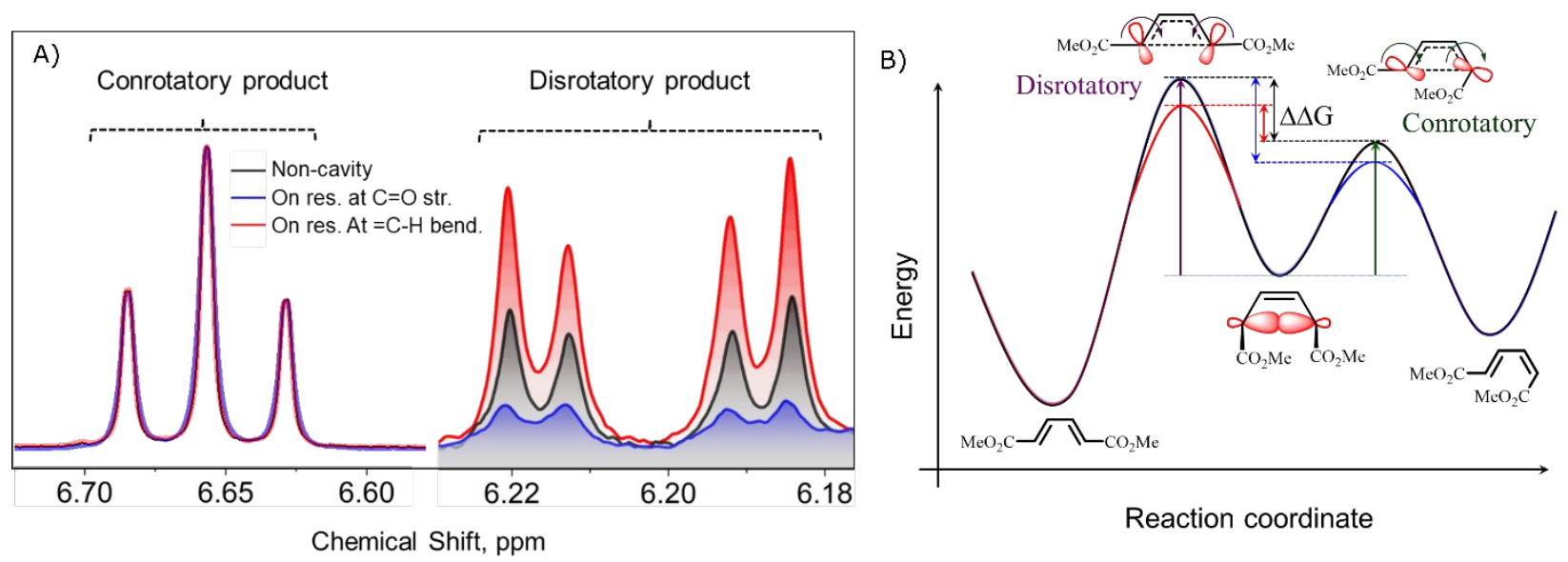

Figure 4. A) NMR quantification of the conrotatory and disrotatory products of CB for noncavity and for VSC of the $\mathrm{C}=\mathrm{O}$ and $\mathrm{C}-\mathrm{H}$ modes. B) Schematic representation of energy level diagram for the con- and disrotatory electrocyclic ring-opening of $\mathrm{CB}$ under non-cavity and VSC conditions. The relative Gibbs free activation energy change between conrotatory and disrotatory transition state is modified depending on the vibration under strong coupling (reproduced with permission from ref. 85).

\section{Theoretical studies}

The theoretical studies of chemistry under VSC are hampered by the fact that sophisticated tools such as those that have been developed in quantum chemistry are necessary to deal with the complexity of even simple chemical reactions. In addition, the interaction with EM field, preferably in a quantified way, needs to be included. Simple models, using JaynesCummings formulation for instance, cannot explain the effects of VSC on chemical reactivity. Several groups have taken up the challenge, leading to further questioning of different explanations but much progress has been made. ${ }^{64,66-68,75,79,81-84,92,94-97}$ For instance, recent $A b$ initio calculations based on quantum electrodynamical density-functional theory has been able tackle the experimentally observed deceleration of PTA deprotection reaction under VSC. ${ }^{95}$ According to this study, VSC plays a major role in redistributing the energy of the bond under strong coupling into other degrees of freedom. As a consequence, the reaction is trapped in its trajectory under VSC and slows down. It is important to note that such an energy redistribution processes are strongly influenced by the symmetry of the vibrations involved. This study also confirms a resonance effect (albeit broader) as seen in all the experimental studies. 


\section{VSC beyond chemical reactivity}

Molecular vibrations and the phonons of solids play important roles in very many material properties from solvation to conductivity. Therefore, there is much room to explore how VSC might perturb or modify material properties. For instance, recently it is shown that the ionic conductivity of the aqueous electrolyte $\mathrm{HClO}_{4}$ is enhanced by an order of magnitude under the VSC of the stretching mode of water. ${ }^{99}$ A few more examples are given below to inspire further work.

\section{Intermolecular interactions and supramolecular assembly}

The vacuum EM fluctuations play a key role in intermolecular interactions such as in the London dispersion forces. Due to the collective character of the polaritonic states, VSC of selective vibrational modes of solvent/molecule should therefore modify their inter- and intramolecular interactions which are the back-bones of clustering, solvation, supramolecular assembly, aggregation and crystallization. For instance, the kinetics and the morphology of supramolecular organization during gelation are significantly modified under VSC; $\frac{100}{12}$ the assembly is found to be flakes under cooperative VSC (with the solvent) when compared to fibers in the non-cavity conditions. On the other hand, Hirai and co-workers investigated the polymorphism in metal organic frameworks crystallization under VSC of solvent molecules. ${ }^{90}$ Preparation of Zeolitic imidazolate framework (ZIF) from aqueous solution of $\mathrm{Zn}\left(\mathrm{NO}_{3}\right)_{2}$ and 2methylimidazole leads to dimorphic structures (ZIF-8 and ZIF-L) under non-cavity conditions. But ZIF-8 crystals are selectively obtained under VSC conditions where the O-H stretching vibration of water is strongly coupled to the cavity vacuum field. Moreover, ZIF crystallization is also accelerated by solvent VSC which could be due to the enhanced solute-solvent interactions under cavity conditions. Theoretical studies also show that intermolecular interactions are modified under electronic strong coupling, ${ }^{97}$ and that self-interaction terms become important in the ultra-strong vibrational coupling regime. ${ }^{55,69,83,94}$

\section{Light induced processes under VSC}

Hybrid polaritonic states, formed under ESC, have proven to show modified excited state properties of the materials such as distance independent long-range energy transfer between the molecules due to their entangled and delocalized nature. ${ }^{18,60}$ Similarly, VSC also alters light 
induced/excited state processes such as vibrational energy transfer. ${ }^{80}$ Pump-probe experiments of polaritonic states under VSC reveal the vibrational dynamics of coupled system and raise further questions about the consequences of VSC on molecular properties. . $^{59,70,89}$ Raman scattering has been reported to be enhanced under both ESC and VSC. ${ }^{52,}{ }^{120-121}$ It is important to note that the enhancement of modified Raman scattering under VSC reported in ref. 52 most likely includes a contribution from a surface enhanced Raman (SERS) effect, in agreement with a more recent study. ${ }^{122}$ We have found that heating by the Raman laser can lead to Au island formation which favors SERS. So great care must be used in probing cavity samples with intense lasers.

\section{Solid state material properties}

Recently, cooperative VSC of phonon modes of superconductors such as YBCO $\left(\mathrm{YBa}_{2} \mathrm{Cu}_{3} \mathrm{O}_{6+\delta}\right)$ and $\mathrm{Rb}_{3} \mathrm{C}_{60}$ with metal ( $\mathrm{Au}$ ) surface plasmon polaritons (SPP) has been reported to modify superconducting transition temperature $\left(\mathrm{T}_{\mathrm{c}}\right){ }^{65}{ }^{65}$ Under such a cooperative VSC, the phonon based superconductor, $\mathrm{Rb}_{3} \mathrm{C}_{60}$ exhibit an increase in $\mathrm{T}_{\mathrm{c}}$ from $30 \mathrm{~K}$ to $45 \mathrm{~K}$ at normal pressures which could be attributed to enhanced electron-phonon coupling under strong coupling condition. For YBCO nanoparticles, Tc decreases from $92 \mathrm{~K}$ to $86 \mathrm{~K}$ is due to competition from a 700-fold enhancement in ferromagnetism when the phonon mode is coupled to SPP modes. The improved ferromagnetic property of YBCO under strong coupling is understood to be due to the collective and extended nature of polaritonic states that enhance the ferromagnetic domains. ${ }^{98}$

\section{Conclusion}

The control of chemical dynamics by using molecular vibrations $\frac{119}{19}$ is an old dream that was explored from the 1980's with the advent of IR lasers. ${ }^{123-126}$ Such physical approach to control the chemical reactivity requires mode selective IR excitation which is efficient only at low temperature due to intra-molecular vibrational relaxation (IVR). VSC offers a novel approach to this quest. No external light source is required and it can be done at room temperature. VSC is easy to implement and the only challenge is to do the experiments in cavities correctly as explained in this perspective.

So far only a handful of different reactions have been reported under VSC. Many more reactions need to be studied in order to find the underlying principles on how VSC affect 22 
chemistry. Different classes of reactions might behave differently under VSC. Cooperative strong coupling facilitates and expands the number of reactions that can be explored. While symmetry plays a fundamental role, there will no doubt be other factors that will come to light as

more coupled systems are studied. Perturbation of solute-solvent interactions under VSC clearly opens a new pathway in the field of chemical equilibria, supramolecular assembly formation and crystal engineering. It is relatively easy to scale up for industrial applications. Thus, VSC will no doubt become a useful tool for chemistry and material science, complementing existing techniques. It will also contribute to the fundamental understanding of the role of vacuum field in common molecular processes and it offers numerous technological applications.

\section{AUTHOR INFORMATION}

\section{Corresponding Authors}

*E-mail: nagarajan@unistra.fr; athomas@iisc.ac.in; ebbesen@unistra.fr

\section{AUTHOR CONTRIBUTIONS}

The manuscript was written through contributions of all authors. All authors have given approval to the final version of the manuscript.

\section{ACKNOWLEDGMENT}

We acknowledge support of the International Center for Frontier Research in Chemistry (icFRC, Strasbourg), the ANR Equipex Union (ANR-10-EQPX-52-01), CSC (ANR-10-LABX- 0026 CSC) within the Investissement d'Avenir program ANR-10-IDEX-0002-02. TWE acknowledges the funding support of the ERC (project no 788482 MOLUSC), QuantERA project RouTe, the European Union's Horizon 2020 research and innovation programme under the Marie Sklodowska-Curie grant No 811284. A.T. thanks IISc for the start-up grant.

The authors declare no competing financial interest. 


\section{References}

[1] Dirac, P. A. M., The Quantum Theory of the Emission and Absorption of Radiation, Proc. R. Soc. Lond. Ser. A Math. Phys. Character 1927, 114, 243-265.

[2] Drexhage, K. H.; Kuhn, H.; Schäfer, F. P., Variation of the Fluorescence Decay Time of a Molecule in Front of a Mirror, Ber. Bunsenges. Phys. Chem. 1968, 72, 329-329.

[3] Drexhage, K. H., Iv Interaction of Light with Monomolecular Dye Layers. In Progress in Optics, Wolf, E., Ed. Elsevier: 1974; Vol. 12, pp 163-232.

[4] Lamb, W. E.; Retherford, R. C., Fine Structure of the Hydrogen Atom by a Microwave Method, Phys. Rev. 1947, 72, 241-243.

[5] Dzyaloshinskii, I. E.; Lifshitz, E. M.; Pitaevskii, L. P., The General Theory of Van Der Waals Forces, Adv. Phys. 1961, 10, 165-209.

[6] Garcia-Vidal, F. J.; Ciuti, C.; Ebbesen, T. W., Manipulating Matter by Strong Coupling to Vacuum Fields, Science 2021, 373, eabd0336.

[7] Hutchison, J. A.; Schwartz, T.; Genet, C.; Devaux, E.; Ebbesen, T. W., Modifying Chemical Landscapes by Coupling to Vacuum Fields, Angew. Chem. Int. Ed. 2012, 51, 1592-1596.

[8] Genet, C.; Faist, J.; Ebbesen, T. W., Inducing New Material Properties with Hybrid LightMatter States, Phys. Today 2021, 74, 42-48.

[9] Ebbesen, T. W., Hybrid Light-Matter States in a Molecular and Material Science Perspective, Acc. Chem. Res. 2016, 49, 2403-2412.

[10] Hutchison, J. A.; Liscio, A.; Schwartz, T.; Canaguier-Durand, A.; Genet, C.; Palermo, V.; Samorì, P.; Ebbesen, T. W., Tuning the Work-Function Via Strong Coupling, Adv. Mater. 2013, 25, 2481-2485.

[11] Orgiu, E.; George, J.; Hutchison, J. A.; Devaux, E.; Dayen, J. F.; Doudin, B.; Stellacci, F.; Genet, C.; Schachenmayer, J.; Genes, C.; Pupillo, G.; Samorì, P.; Ebbesen, T. W., Conductivity in Organic Semiconductors Hybridized with the Vacuum Field, Nat. Mater. 2015, 14, 1123-1129.

[12] Zeng, P.; Cadusch, J.; Chakraborty, D.; Smith, T. A.; Roberts, A.; Sader, J. E.; Davis, T. J.; Gómez, D. E., Photoinduced Electron Transfer in the Strong Coupling Regime: Waveguide-Plasmon Polaritons, Nano Lett. 2016, 16, 2651-2656.

[13] Paravicini-Bagliani, G. L.; Appugliese, F.; Richter, E.; Valmorra, F.; Keller, J.; Beck, M.; Bartolo, N.; Rössler, C.; Ihn, T.; Ensslin, K.; Ciuti, C.; Scalari, G.; Faist, J., MagnetoTransport Controlled by Landau Polariton States, Nat. Phys. 2019, 15, 186-190.

[14] Krainova, N.; Grede, A. J.; Tsokkou, D.; Banerji, N.; Giebink, N. C., Polaron Photoconductivity in the Weak and Strong Light-Matter Coupling Regime, Phys. Rev. Lett. 2020, 124, 177401.

[15] Nagarajan, K.; George, J.; Thomas, A.; Devaux, E.; Chervy, T.; Azzini, S.; Joseph, K.; Jouaiti, A.; Hosseini, M. W.; Kumar, A.; Genet, C.; Bartolo, N.; Ciuti, C.; Ebbesen, T. W., Conductivity and Photoconductivity of a p-Type Organic Semiconductor under Ultrastrong Coupling, ACS Nano 2020, 14, 10219-10225. 
[16] Coles, D. M.; Somaschi, N.; Michetti, P.; Clark, C.; Lagoudakis, P. G.; Savvidis, P. G.; Lidzey, D. G., Polariton-Mediated Energy Transfer between Organic Dyes in A Strongly Coupled Optical Microcavity, Nat. Mater. 2014, 13, 712-719.

[17] Zhong, X.; Chervy, T.; Wang, S.; George, J.; Thomas, A.; Hutchison, J. A.; Devaux, E.; Genet, C.; Ebbesen, T. W., Non-Radiative Energy Transfer Mediated by Hybrid LightMatter States, Angew. Chem. Int. Ed. 2016, 55, 6202-6206.

[18] Zhong, X.; Chervy, T.; Zhang, L.; Thomas, A.; George, J.; Genet, C.; Hutchison, J. A.; Ebbesen, T. W., Energy Transfer between Spatially Separated Entangled Molecules, Angew. Chem. Int. Ed. 2017, 56, 9034-9038.

[19] Akulov, K.; Bochman, D.; Golombek, A.; Schwartz, T., Long-Distance Resonant Energy Transfer Mediated by Hybrid Plasmonic-Photonic Modes, J. Phys. Chem. C 2018, 122, 15853-15860.

[20] Rozenman, G. G.; Akulov, K.; Golombek, A.; Schwartz, T., Long-Range Transport of Organic Exciton-Polaritons Revealed by Ultrafast Microscopy, ACS Photonics 2018, 5, 105-110.

[21] Hou, S.; Khatoniar, M.; Ding, K.; Qu, Y.; Napolov, A.; Menon, V. M.; Forrest, S. R., Ultralong-Range Energy Transport in a Disordered Organic Semiconductor at Room Temperature Via Coherent Exciton-Polariton Propagation, Adv. Mater. 2020, 32, 2002127.

[22] Stranius, K.; Hertzog, M.; Börjesson, K., Selective Manipulation of Electronically Excited States through Strong Light-Matter Interactions, Nat. Commun. 2018, 9, 2273.

[23] Eizner, E.; Martínez-Martínez, L. A.; Yuen-Zhou, J.; Kéna-Cohen, S., Inverting Singlet and Triplet Excited States Using Strong Light-Matter Coupling, Sci. Adv. 2019, 5, eaax4482.

[24] Ye, C.; Mallick, S.; Hertzog, M.; Kowalewski, M.; Börjesson, K., Direct Transition from Triplet Excitons to Hybrid Light-Matter States Via Triplet-Triplet Annihilation, J. Am. Chem. Soc. 2021, 143, 7501-7508.

[25] Chervy, T.; Xu, J.; Duan, Y.; Wang, C.; Mager, L.; Frerejean, M.; Münninghoff, J. A. W.; Tinnemans, P.; Hutchison, J. A.; Genet, C.; Rowan, A. E.; Rasing, T.; Ebbesen, T. W., High-Efficiency Second-Harmonic Generation from Hybrid Light-Matter States, Nano Lett. 2016, 16, 7352-7356.

[26] Barachati, F.; Simon, J.; Getmanenko, Y. A.; Barlow, S.; Marder, S. R.; Kéna-Cohen, S., Tunable Third-Harmonic Generation from Polaritons in the Ultrastrong Coupling Regime, ACS Photonics 2018, 5, 119-125.

[27] Liu, B.; Crescimanno, M.; Twieg, R. J.; Singer, K. D., Dispersion of Third-Harmonic Generation in Organic Cavity Polaritons, Adv. Opt. Mater. 2019, 7, 1801682.

[28] Wang, K.; Seidel, M.; Nagarajan, K.; Chervy, T.; Genet, C.; Ebbesen, T., Large Optical Nonlinearity Enhancement under Electronic Strong Coupling, Nat. Commun. 2021, 12, 1486.

[29] Munkhbat, B.; Wersäll, M.; Baranov, D. G.; Antosiewicz, T. J.; Shegai, T., Suppression of Photo-Oxidation of Organic Chromophores by Strong Coupling to Plasmonic Nanoantennas, Sci. Adv. 2018, 4, eaas9552. 
[30] Peters, V. N.; Faruk, M. O.; Asane, J.; Alexander, R.; Peters, D. a. A.; Prayakarao, S.; Rout, S.; Noginov, M. A., Effect of Strong Coupling on Photodegradation of the Semiconducting Polymer P3ht, Optica 2019, 6, 318-325.

[31] Galego, J.; Garcia-Vidal, F. J.; Feist, J., Cavity-Induced Modifications of Molecular Structure in the Strong-Coupling Regime, Phys. Rev. X 2015, 5, 041022.

[32] Feist, J.; Garcia-Vidal, F. J., Extraordinary Exciton Conductance Induced by Strong Coupling, Phys. Rev. Lett. 2015, 114, 196402.

[33] Gonzalez-Ballestero, C.; Feist, J.; Moreno, E.; Garcia-Vidal, F. J., Harvesting Excitons through Plasmonic Strong Coupling, Phys. Rev. B 2015, 92, 121402.

[34] Schachenmayer, J.; Genes, C.; Tignone, E.; Pupillo, G., Cavity-Enhanced Transport of Excitons, Phys. Rev. Lett. 2015, 114, 196403.

[35] Galego, J.; Garcia-Vidal, F. J.; Feist, J., Suppressing Photochemical Reactions with Quantized Light Fields, Nat. Commun. 2016, 7, 13841.

[36] Herrera, F.; Spano, F. C., Cavity-Controlled Chemistry in Molecular Ensembles, Phys. Rev. Lett. 2016, 116, 238301.

[37] Hagenmüller, D.; Schachenmayer, J.; Schütz, S.; Genes, C.; Pupillo, G., Cavity-Enhanced Transport of Charge, Phys. Rev. Lett. 2017, 119, 223601.

[38] Flick, J.; Ruggenthaler, M.; Appel, H.; Rubio, A., Atoms and Molecules in Cavities, from Weak to Strong Coupling in Quantum-Electrodynamics (QED) Chemistry, Proc. Natl. Acad. Sci. U. S. A.. 2017, 114, 3026-3034.

[39] Feist, J.; Galego, J.; Garcia-Vidal, F. J., Polaritonic Chemistry with Organic Molecules, ACS Photonics 2018, 5, 205-216.

[40] Bartolo, N.; Ciuti, C., Vacuum-Dressed Cavity Magnetotransport of A Two-Dimensional Electron Gas, Phys. Rev. B 2018, 98, 205301.

[41] Flick, J.; Schäfer, C.; Ruggenthaler, M.; Appel, H.; Rubio, A., Ab Initio Optimized Effective Potentials for Real Molecules in Optical Cavities: Photon Contributions to the Molecular Ground State, ACS Photonics 2018, 5, 992-1005.

[42] Szidarovszky, T.; Halász, G. J.; Császár, A. G.; Cederbaum, L. S.; Vibók, Á., Conical Intersections Induced by Quantum Light: Field-Dressed Spectra from the Weak to the Ultrastrong Coupling Regimes, J. Phys. Chem. Lett. 2018, 9, 6215-6223.

[43] Groenhof, G.; Toppari, J. J., Coherent Light Harvesting through Strong Coupling to Confined Light, J. Phys. Chem. Lett. 2018, 9, 4848-4851.

[44] Martínez-Martínez, L. A.; Ribeiro, R. F.; Campos-González-Angulo, J.; Yuen-Zhou, J., Can Ultrastrong Coupling Change Ground-State Chemical Reactions?, ACS Photonics 2018, 5, 167-176.

[45] Schäfer, C.; Ruggenthaler, M.; Appel, H.; Rubio, A., Modification of Excitation and Charge Transfer in Cavity Quantum-Electrodynamical Chemistry, Proc. Natl. Acad. Sci. U. S. A.. 2019, 116, 4883-4892.

[46] Fregoni, J.; Granucci, G.; Persico, M.; Corni, S., Strong Coupling with Light Enhances the Photoisomerization Quantum Yield of Azobenzene, Chem 2020, 6, 250-265.

[47] Gu, B.; Mukamel, S., Cooperative Conical Intersection Dynamics of Two Pyrazine Molecules in an Optical Cavity, J. Phys. Chem. Lett. 2020, 11, 5555-5562. 
[48] Herrera, F.; Owrutsky, J., Molecular Polaritons for Controlling Chemistry with Quantum Optics, J. Chem. Phys. 2020, 152, 100902.

[49] Thomas, A.; George, J.; Shalabney, A.; Dryzhakov, M.; Varma, S. J.; Moran, J.; Chervy, T.; Zhong, X.; Devaux, E.; Genet, C.; Hutchison, J. A.; Ebbesen, T. W., Ground-State Chemical Reactivity under Vibrational Coupling to the Vacuum Electromagnetic Field, Angew. Chem. Int. Ed. 2016, 55, 11462-11466.

[50] Shalabney, A.; George, J.; Hutchison, J.; Pupillo, G.; Genet, C.; Ebbesen, T. W., Coherent Coupling of Molecular Resonators with A Microcavity Mode, Nat. Commun. 2015, 6, 5981.

[51] Long, J. P.; Simpkins, B. S., Coherent Coupling between a Molecular Vibration and Fabry-Perot Optical Cavity to Give Hybridized States in the Strong Coupling Limit, ACS Photonics 2015, 2, 130-136.

[52] Shalabney, A.; George, J.; Hiura, H.; Hutchison, J. A.; Genet, C.; Hellwig, P.; Ebbesen, T. W., Enhanced Raman Scattering from Vibro-Polariton Hybrid States, Angew. Chem. Int. Ed. 2015, 54, 7971-7975.

[53] George, J.; Shalabney, A.; Hutchison, J. A.; Genet, C.; Ebbesen, T. W., Liquid-Phase Vibrational Strong Coupling, J. Phys. Chem. Lett. 2015, 6, 1027-1031.

[54] Dunkelberger, A. D.; Spann, B. T.; Fears, K. P.; Simpkins, B. S.; Owrutsky, J. C., Modified Relaxation Dynamics and Coherent Energy Exchange in Coupled VibrationCavity Polaritons, Nat. Commun. 2016, 7, 13504.

[55] George, J.; Chervy, T.; Shalabney, A.; Devaux, E.; Hiura, H.; Genet, C.; Ebbesen, T. W., Multiple Rabi Splittings under Ultrastrong Vibrational Coupling, Phys. Rev. Lett. 2016, $117,153601$.

[56] Vergauwe, R. M. A.; George, J.; Chervy, T.; Hutchison, J. A.; Shalabney, A.; Torbeev, V. Y.; Ebbesen, T. W., Quantum Strong Coupling with Protein Vibrational Modes, J. Phys. Chem. Lett. 2016, 7, 4159-4164.

[57] Muallem, M.; Palatnik, A.; Nessim, G. D.; Tischler, Y. R., Strong Light-Matter Coupling and Hybridization of Molecular Vibrations in a Low-Loss Infrared Microcavity, J. Phys. Chem. Lett. 2016, 7, 2002-2008.

[58] Casey, S. R.; Sparks, J. R., Vibrational Strong Coupling of Organometallic Complexes, J. Phys. Chem. C 2016, 120, 28138-28143.

[59] Xiang, B.; Ribeiro, R. F.; Dunkelberger, A. D.; Wang, J.; Li, Y.; Simpkins, B. S.; Owrutsky, J. C.; Yuen-Zhou, J.; Xiong, W., Two-Dimensional Infrared Spectroscopy of Vibrational Polaritons, Proc. Natl. Acad. Sci. U. S. A.. 2018, 115, 4845-4850.

[60] Sáez-Blázquez, R.; Feist, J.; Fernández-Domínguez, A. I.; García-Vidal, F. J., Organic Polaritons Enable Local Vibrations to Drive Long-Range Energy Transfer, Phys. Rev. B 2018, 97, 241407.

[61] Vergauwe, R. M. A.; Thomas, A.; Nagarajan, K.; Shalabney, A.; George, J.; Chervy, T.; Seidel, M.; Devaux, E.; Torbeev, V.; Ebbesen, T. W., Modification of Enzyme Activity by Vibrational Strong Coupling of Water, Angew. Chem. Int. Ed. 2019, 58, 15324-15328.

[62] Lather, J.; Bhatt, P.; Thomas, A.; Ebbesen, T. W.; George, J., Cavity Catalysis by Cooperative Vibrational Strong Coupling of Reactant and Solvent Molecules, Angew. Chem. Int. Ed. 2019, 58, 10635-10638. 
[63] Thomas, A.; Lethuillier-Karl, L.; Nagarajan, K.; Vergauwe, R. M. A.; George, J.; Chervy, T.; Shalabney, A.; Devaux, E.; Genet, C.; Moran, J.; Ebbesen, T. W., Tilting A GroundState Reactivity Landscape by Vibrational Strong Coupling, Science 2019, 363, 615-619.

[64] Campos-Gonzalez-Angulo, J. A.; Ribeiro, R. F.; Yuen-Zhou, J., Resonant Catalysis of Thermally Activated Chemical Reactions with Vibrational Polaritons, Nat. Commun. 2019, 10, 4685.

[65] Thomas, A.; Devaux, E.; Nagarajan, K.; Chervy, T.; Seidel, M.; Hagenmüller, D.; Schütz, S.; Schachenmayer, J.; Genet, C.; Pupillo, G.; Ebbesen, T. W., Exploring Superconductivity under Strong Coupling with the Vacuum Electromagnetic Field, ArXiv 2019, arXiv:1911.01459v2.

[66] Du, M.; Ribeiro, R. F.; Yuen-Zhou, J., Remote Control of Chemistry in Optical Cavities, Chem 2019, 5, 1167-1181.

[67] Hernández, F. J.; Herrera, F., Multi-Level Quantum Rabi Model for Anharmonic Vibrational Polaritons, J. Chem. Phys. 2019, 151, 144116.

[68] Galego, J.; Climent, C.; Garcia-Vidal, F. J.; Feist, J., Cavity Casimir-Polder Forces and Their Effects in Ground-State Chemical Reactivity, Phys. Rev. X 2019, 9, 021057.

[69] Damari, R.; Weinberg, O.; Krotkov, D.; Demina, N.; Akulov, K.; Golombek, A.; Schwartz, T.; Fleischer, S., Strong Coupling of Collective Intermolecular Vibrations in Organic Materials at Terahertz Frequencies, Nat. Commun. 2019, 10, 3248.

[70] Xiang, B.; Ribeiro, R. F.; Li, Y.; Dunkelberger, A. D.; Simpkins, B. B.; Yuen-Zhou, J.; Xiong, W., Manipulating Optical Nonlinearities of Molecular Polaritons by Delocalization, Sci. Adv. 2019, 5, eaax5196.

[71] Hirai, K.; Hutchison, J. A.; Uji-i, H., Recent Progress in Vibropolaritonic Chemistry, ChemPlusChem 2020, 85, 1981-1988.

[72] Schütz, S.; Schachenmayer, J.; Hagenmüller, D.; Brennen, G. K.; Volz, T.; Sandoghdar, V.; Ebbesen, T. W.; Genes, C.; Pupillo, G., Ensemble-Induced Strong Light-Matter Coupling of a Single Quantum Emitter, Phys. Rev. Lett. 2020, 124, 113602.

[73] Takele, W. M.; Wackenhut, F.; Piatkowski, L.; Meixner, A. J.; Waluk, J., Multimode Vibrational Strong Coupling of Methyl Salicylate to a Fabry-Pérot Microcavity, J. Phys. Chem. B 2020, 124, 5709-5716.

[74] Scholes, G. D.; DelPo, C. A.; Kudisch, B., Entropy Reorders Polariton States, J. Phys. Chem. Lett. 2020, 11, 6389-6395.

[75] Vurgaftman, I.; Simpkins, B. S.; Dunkelberger, A. D.; Owrutsky, J. C., Negligible Effect of Vibrational Polaritons on Chemical Reaction Rates Via the Density of States Pathway, J. Phys. Chem. Lett. 2020, 11, 3557-3562.

[76] Hirai, K.; Takeda, R.; Hutchison, J. A.; Uji-i, H., Modulation of Prins Cyclization by Vibrational Strong Coupling, Angew. Chem. Int. Ed. 2020, 59, 5332-5335.

[77] Pang, Y.; Thomas, A.; Nagarajan, K.; Vergauwe, R. M. A.; Joseph, K.; Patrahau, B.; Wang, K.; Genet, C.; Ebbesen, T. W., On the Role of Symmetry in Vibrational Strong Coupling: The Case of Charge-Transfer Complexation, Angew. Chem. Int. Ed. 2020, 59, 10436-10440.

[78] Thomas, A.; Jayachandran, A.; Lethuillier-Karl, L.; Vergauwe, R. M. A.; Nagarajan, K.; Devaux, E.; Genet, C.; Moran, J.; Ebbesen, T. W., Ground State Chemistry under 
Vibrational Strong Coupling: Dependence of Thermodynamic Parameters on the Rabi Splitting Energy, Nanophotonics 2020, 9, 249-255.

[79] Zhdanov, V. P., Vacuum Field in A Cavity, Light-Mediated Vibrational Coupling, and Chemical Reactivity, Chem. Phys. 2020, 535, 110767.

[80] Xiang, B.; Ribeiro, R. F.; Du, M.; Chen, L.; Yang, Z.; Wang, J.; Yuen-Zhou, J.; Xiong, W., Intermolecular Vibrational Energy Transfer Enabled by Microcavity Strong Light-Matter Coupling, Science 2020, 368, 665-667.

[81] Phuc, N. T.; Trung, P. Q.; Ishizaki, A., Controlling the Nonadiabatic Electron-Transfer Reaction Rate through Molecular-Vibration Polaritons in the Ultrastrong Coupling Regime, Sci. Rep. 2020, 10, 7318.

[82] Triana, J.; Herrera, F., Self-Dissociation of Polar Molecules in a Confined Infrared Vacuum, ChemRxiv 2020, 10.26434/chemrxiv.12702419.v1.

[83] Li, T. E.; Subotnik, J. E.; Nitzan, A., Cavity Molecular Dynamics Simulations of Liquid Water under Vibrational Ultrastrong Coupling, Proc. Natl. Acad. Sci. U. S. A.. 2020, 117, 18324-18331.

[84] Li, X.; Mandal, A.; Huo, P., Cavity Frequency-Dependent Theory for Vibrational Polariton Chemistry, Nat. Commun. 2021, 12, 1315.

[85] Sau, A.; Nagarajan, K.; Patrahau, B.; Lethuillier-Karl, L.; Vergauwe, R. M. A.; Thomas, A.; Moran, J.; Genet, C.; Ebbesen, T. W., Modifying Woodward-Hoffmann Stereoselectivity under Vibrational Strong Coupling, Angew. Chem. Int. Ed. 2021, 60, 5712-5717.

[86] Lather, J.; George, J., Improving Enzyme Catalytic Efficiency by Co-Operative Vibrational Strong Coupling of Water, J. Phys. Chem. Lett. 2021, 12, 379-384.

[87] Hiura, H.; Shalabney, A., Vacuum-Field Catalysis: Accelerated Reactions by Vibrational Ultra Strong Coupling, ChemRxiv 2021, 10.26434/chemrxiv.7234721.v5.

[88] Lather, J.; K, T. A. N.; Singh, J.; George, J., Cavity Catalysis: Modifying Linear FreeEnergy Relationship under Cooperative Vibrational Strong Coupling, ChemRxiv 2021, 10.33774/chemrxiv-2021-97v8x.

[89] Grafton, A. B.; Dunkelberger, A. D.; Simpkins, B. S.; Triana, J. F.; Hernández, F. J.; Herrera, F.; Owrutsky, J. C., Excited-State Vibration-Polariton Transitions and Dynamics in Nitroprusside, Nat. Commun. 2021, 12, 214.

[90] Hirai, K.; Ishikawa, H.; Chervy, T.; Hutchison, J. A.; Uji-i, H., Selective Crystallization Via Vibrational Strong Coupling, Chem. Sci. 2021, DOI: 10.1039/D1SC03706D.

[91] Imperatore, M. V.; Asbury, J. B.; Giebink, N. C., Reproducibility of Cavity-Enhanced Chemical Reaction Rates in the Vibrational Strong Coupling Regime, J. Chem. Phys. 2021, 154, 191103.

[92] Fischer, E. W.; Saalfrank, P., Ground State Properties and Infrared Spectra of Anharmonic Vibrational Polaritons of Small Molecules in Cavities, J. Chem. Phys. 2021, 154, 104311.

[93] Xiang, B.; Wang, J.; Yang, Z.; Xiong, W., Nonlinear Infrared Polaritonic Interaction between Cavities Mediated by Molecular Vibrations at Ultrafast Time Scale, Sci. Adv. 2021, 7, eabf6397.

[94] Kadyan, A.; Shaji, A.; George, J., Boosting Self-Interaction of Molecular Vibrations under Ultrastrong Coupling Condition, J. Phys. Chem. Lett. 2021, 12, 4313-4318. 
[95] Schäfer, C.; Flick, J.; Ronca, E.; Narang, P.; Rubio, A., Shining Light on the Microscopic Resonant Mechanism Responsible for Cavity-Mediated Chemical Reactivity, ArXiv 2021, arXiv:2104.12429v2.

[96] Li, T. E.; Nitzan, A.; Subotnik, J. E., Collective Vibrational Strong Coupling Effects on Molecular Vibrational Relaxation and Energy Transfer: Numerical Insights Via Cavity Molecular Dynamics Simulations, Angew. Chem. Int. Ed. 2021, 60, 15533-15540.

[97] Haugland, T. S.; Schäfer, C.; Ronca, E.; Rubio, A.; Koch, H., Intermolecular Interactions in Optical Cavities: An Ab Initio QED Study, J. Chem. Phys. 2021, 154, 094113.

[98] Thomas, A.; Devaux, E.; Nagarajan, K.; Rogez, G.; Seidel, M.; Richard, F.; Genet, C.; Drillon, M.; Ebbesen, T. W., Large Enhancement of Ferromagnetism under a Collective Strong Coupling of YBCO Nanoparticles, Nano Lett. 2021, 21, 4365-4370.

[99] Fukushima, T.; Yoshimitsu, S.; Murakoshi, K., Inherent Promotion of Ionic Conductivity Via Coherent Vibrational Strong Coupling of Water, ChemRxiv 2021, 10.33774/chemrxiv2021-zk549.

[100] Joseph, K.; Kushida, S.; Smarsly, E.; Ihiawakrim, D.; Thomas, A.; Paravicini-Bagliani, G. L.; Nagarajan, K.; Vergauwe, R.; Devaux, E.; Ersen, O.; Bunz, U. H. F.; Ebbesen, T. W., Supramolecular Assembly of Conjugated Polymers under Vibrational Strong Coupling, Angew. Chem. Int. Ed. 2021, 60, 19665-19670.

[101] Purcell, E. M., Spontaneous Emission Probabilities at Radio Frequencies, Phys. Rev. 1946, 69, 681.

[102] Hestand, N. J.; Spano, F. C., Molecular Aggregate Photophysics Beyond the Kasha Model: Novel Design Principles for Organic Materials, Acc. Chem. Res. 2017, 50, 341-350.

[103] Aberra Guebrou, S.; Symonds, C.; Homeyer, E.; Plenet, J. C.; Gartstein, Y. N.; Agranovich, V. M.; Bellessa, J., Coherent Emission from a Disordered Organic Semiconductor Induced by Strong Coupling with Surface Plasmons, Phys. Rev. Lett. 2012, 108, 066401.

[104] Shi, L.; Hakala, T. K.; Rekola, H. T.; Martikainen, J. P.; Moerland, R. J.; Törmä, P., Spatial Coherence Properties of Organic Molecules Coupled to Plasmonic Surface Lattice Resonances in the Weak and Strong Coupling Regimes, Phys. Rev. Lett. 2014, 112, 153002.

[105] Wang, S.; Chervy, T.; George, J.; Hutchison, J. A.; Genet, C.; Ebbesen, T. W., Quantum Yield of Polariton Emission from Hybrid Light-Matter States, J. Phys. Chem. Lett. 2014, 5, 1433-1439.

[106] Canaguier-Durand, A.; Genet, C.; Lambrecht, A.; Ebbesen, T. W.; Reynaud, S., NonMarkovian Polariton Dynamics in Organic Strong Coupling, Eur. Phys. J. D 2015, 69, 24.

[107] Putz, S.; Krimer, D. O.; Amsüss, R.; Valookaran, A.; Nöbauer, T.; Schmiedmayer, J.; Rotter, S.; Majer, J., Protecting a Spin Ensemble against Decoherence in the StrongCoupling Regime of Cavity QED, Nat. Phys. 2014, 10, 720-724.

[108] Chiang, K.-T.; Zhang, W.-M., Non-Markovian Decoherence Dynamics of Strong-Coupling Hybrid Quantum Systems: A Master Equation Approach, Physical Review A 2021, 103, 013714.

[109] Ciuti, C.; Bastard, G.; Carusotto, I., Quantum Vacuum Properties of the Intersubband Cavity Polariton Field, Phys. Rev. B 2005, 72, 115303. 
[110] Frisk Kockum, A.; Miranowicz, A.; De Liberato, S.; Savasta, S.; Nori, F., Ultrastrong Coupling between Light and Matter, Nat. Rev. Phys. 2019, 1, 19-40.

[111] Menghrajani, K. S.; Nash, G. R.; Barnes, W. L., Vibrational Strong Coupling with Surface Plasmons and the Presence of Surface Plasmon Stop Bands, ACS Photonics 2019, 6, 21102116.

[112] Dayal, G.; Morichika, I.; Ashihara, S., Vibrational Strong Coupling in Subwavelength Nanogap Patch Antenna at the Single Resonator Level, J. Phys. Chem. Lett. 2021, 12, 3171-3175.

[113] Menghrajani, K. S.; Barnes, W. L., Strong Coupling Beyond the Light-Line, ACS Photonics 2020, 7, 2448-2459.

[114] Tutorial on Vsc. Vol. https://seafile.unistra.fr/d/7bb78e5a4607424f94b5/.

[115] Schwartz, T.; Hutchison, J. A.; Léonard, J.; Genet, C.; Haacke, S.; Ebbesen, T. W., Polariton Dynamics under Strong Light-Molecule Coupling, ChemPhysChem 2013, 14, 125-131.

[116] Georgiou, K.; Jayaprakash, R.; Lidzey, D. G., Strong Coupling of Organic Dyes Located at the Surface of a Dielectric Slab Microcavity, J. Phys. Chem. Lett. 2020, 11, 9893-9900.

[117] Woodward, R. B.; Hoffmann, R., The Conservation of Orbital Symmetry, Angew. Chem. Int. Ed. 1969, 8, 781-853.

[118] Fukui, K., Recognition of Stereochemical Paths by Orbital Interaction, Acc. Chem. Res. 1971, 4, 57-64.

[119] Bader, R. F. W., Vibrationally Induced Perturbations in Molecular Electron Distributions, Can. J. Chem. 1962, 40, 1164-1175.

[120] Fainstein, A.; Jusserand, B.; Thierry-Mieg, V., Cavity-Polariton Mediated Resonant Raman Scattering, Phys. Rev. Lett. 1997, 78, 1576-1579.

[121] Tartakovskii, A. I.; Emam-Ismail, M.; Lidzey, D. G.; Skolnick, M. S.; Bradley, D. D. C.; Walker, S.; Agranovich, V. M., Raman Scattering in Strongly Coupled Organic Semiconductor Microcavities, Phys. Rev. B 2001, 63, 121302.

[122] Takele, W. M.; Piatkowski, L.; Wackenhut, F.; Gawinkowski, S.; Meixner, A. J.; Waluk, J., Scouting for Strong Light-Matter Coupling Signatures in Raman Spectra, Phys. Chem. Chem. Phys. 2021, 23, 16837-16846.

[123] Frei, H.; Fredin, L.; Pimentel, G. C., Vibrational Excitation of Ozone and Molecular Fluorine Reactions in Cryogenic Matrices, J. Chem. Phys. 1981, 74, 397-411.

[124] Sinha, A.; Hsiao, M. C.; Crim, F. F., Controlling Bimolecular Reactions: Mode and Bond Selected Reaction of Water with Hydrogen Atoms, J. Chem. Phys. 1991, 94, 4928-4935.

[125] Zare, R. N., Laser Control of Chemical Reactions, Science 1998, 279, 1875-1879.

[126] Crim, F. F., Vibrational State Control of Bimolecular Reactions: Discovering and Directing the Chemistry, Acc. Chem. Res. 1999, 32, 877-884. 